\title{
Preface: Use of NK Cells in Cancer Immunotherapy: Successes and Challenges
}

NK cell biologists have been studying these cells tirelessly since their identification in the 1970s. We have learned a wealth of knowledge regarding the phenotypic and functional attributes of these important cells, as well as the nature of the cells with which they interact, and how their function is modulated in health and disease.

The identification of CSCs or poorly differentiated tumors as targets of NK cells has finally shown the indispensable role these effectors play in successful therapy. In addition, many previous studies have demonstrated the dysfunctional nature of NK cells in cancer patients, indicating that successful therapy will require the restoration of NK cell function in cancer patients. As a consequence of optimal functioning of NK cells in the tumor microenvironment, it is likely that other cancer therapeutic strategies such as chemotherapy and radiotherapy will be more effective in eliminating tumors in cancer patients. Due to the suppressive nature of the tumor microenvironment and systemic immunosuppression, it is important to understand the mechanisms by which the suppressive cells induce inactivation in NK cells. In addition, strategies should be designed to allow maintenance of good NK expansion and function in vivo in cancer patients since NK cells are important in expanding other important cells such as cancer specific $\mathrm{CD} 8^{+} \mathrm{T}$ cells, while limiting the expansion of TAMs, Tregs, MDSCs, MSCs, and fibroblasts, which are the suppressors of $\mathrm{NK}$ and $\mathrm{CD} 8^{+} \mathrm{T}$ cells and thus are hallmarks of aggressive tumors.

In this collection of papers, Drs. Wang, Uyemura, Hashemi, Bjorgaard, Riese, Verbsky, Thakar, and Malarkannan review the role of GATA2, a master regulator that drives the commitment of common lymphoid progenitors (CLPs) into immature NK progenitors (NKPs). They further discuss the outcome of GATA2 haploinsufficiency in patients resulting in the loss of CD56 $6^{\text {bright }} \mathrm{NK}$ cells. Therefore, the review focuses on the recent progress in understanding GATA2 role in human NK cell development and functions.
Drs. Hashemi, Bjorgaard, Wang, Uyemura, Riese, Thakar, and Malarkannan review the recent studies in hematopoietic dysfunction, focusing on impairment in the development and functions of NK cells in patients with Fanconi anemia, and discuss the possible mechanisms of such dysfunction and provide perspectives on future directions.

Drs. Touboul, Zaravinos, and Bonavida review the role of NK cells in skin melanoma. Additionally, they discuss the dysregulation of the activating receptor NKG2D in NK cells in patients, since NKG2D is one of the important receptors in regulating the cytotoxic function and cytokine secretion capabilities of these cells. Using bioinformatics, they confirm the low transcript levels of NKG2D in patients and discuss the potential therapeutic approaches that can be exploited to upregulate NKG2D in patients' NK cells to restore their anti-tumor effects, resulting in increased survival of patients.

Dr. Wu reviews the clinical significance of NK cell therapy in controlling metastatic prostate cancer and provides perspectives on harnessing the function of NK cells in treating metastatic castration-resistant prostate cancer (mCRPC). In addition, she provides insights regarding the role of NK cells in converting the relatively cold mCRPC to hot tumors.

Drs. Jakoš, Pišlar, Jewett, and Kos review the role of MDSCs in inactivation of $\mathrm{NK}$ and $\mathrm{CD} 8^{+}$ $\mathrm{T}$ cells. MDSCs utilize numerous mechanisms to suppress the functions of cytotoxic $\mathrm{T}$ lymphocytes and NK cells, including peptidases. MDSC-derived peptidases inactivate the function of NK cells by triggering ligand shedding for NK cell activating receptors. Moreover, they promote MDSC accumulation, expansion, and survival. They further discuss the multifaceted nature of peptidases relevant to tumors as well as to the functioning of the immune cells.

Drs. Kaur, Ko, Chen, Breznik, Senjor, Wong, Wang, Chovatiya, and Jewett review the timely topic 
of probiotics and their role on the expansion and function of NK cells. They highlight the current understanding of the role of probiotics in enhancement of cancer therapeutics. Moreover, they discuss the role of probiotics in reversal of functional impairment of NK cells. They suggest that NK cell-based immunotherapies in combination with well-selected strains of probiotic bacteria might represent one of the best adjunct therapeutic approaches to prevent and treat cancer in the future.

Guest Editor:

Anahid Jewett

UCLA School of Dentistry and Medicine, Los Angeles, CA, USA 